\title{
LOWER VOLUME GROWTH ESTIMATES FOR SELF-SHRINKERS OF MEAN CURVATURE FLOW
}

\author{
HAIZHONG LI AND YONG WEI
}

(Communicated by Lei Ni)

\begin{abstract}
We obtain a Calabi-Yau type volume growth estimate for complete noncompact self-shrinkers of the mean curvature flow. More precisely, every complete noncompact properly immersed self-shrinker has at least linear volume growth.
\end{abstract}

\section{INTRODUCTION}

On a complete noncompact Riemannian manifold $M^{n}$ with nonnegative Ricci curvature, there are two well-known theorems on volume growth estimates of geodesic balls. One is the classic Bishop volume comparison theorem (see [12, 16]), which says the geodesic balls have at most Euclidean growth; i.e., there exists some positive constant $C$ such that

$$
\operatorname{Vol}\left(B_{x_{0}}(r)\right) \leq C r^{n}
$$

holds for $r>0$ sufficiently large. The other is a theorem proved by Calabi [1] and Yau 18 independently, which says the geodesic balls of such manifolds have at least linear volume growth; that is,

$$
\operatorname{Vol}\left(B_{x_{0}}(r)\right) \geq C r
$$

holds for some positive constant $C$.

In this paper, we consider the volume growth estimates on self-shrinkers. Note that there are many similarities between self-shrinkers and gradient shrinking solitons. Self-shrinkers give homothetically self-shrinking solutions to mean curvature flow and describe possible blow ups at a given singularity of the mean curvature flow. While gradient shrinking Ricci solitons also correspond to the self-similar solutions of Hamilton's Ricci flow, they often arise as Type I singularity models.

Before we state our main theorem, we would like to give a rough brief review about the already known results on volume growth of gradient shrinking Ricci solitons and self-shrinkers.

For an $n$-dimensional complete noncompact gradient shrinking Ricci soliton $(M, g, f)$ satisfying

$$
R_{i j}+f_{i j}=\frac{1}{2} g_{i j}
$$

Received by the editors May 18, 2012 and, in revised form, September 18, 2012. 2010 Mathematics Subject Classification. Primary 53C44; Secondary 53C42.

Key words and phrases. Self-shrinker, volume growth estimate, mean curvature flow.

The authors were supported by NSFC No. 11271214 and Tsinghua University-K.U. Leuven Bilateral Scientific Cooperation Fund. 
H.-D. Cao and D. Zhou 4 proved that it has at most Euclidean volume growth (see also [7, [19]). On the lower volume growth estimate, H.-D. Cao and X. P. Zhu [2] proved that any complete noncompact gradient shrinking Ricci soliton must have infinite volume. In fact, they showed that there is some positive constant $C$ such that $\operatorname{Vol}\left(B_{x_{0}}(r)\right) \geq C \ln \ln r$ for $r$ sufficiently large. If the Ricci curvature is bounded, Carillo and $\mathrm{Ni}[6$ showed that the volume grows at least linearly. If the average scalar curvature satisfies

$$
\frac{1}{\operatorname{Vol}(B(r))} \int_{B(r)} R d v \leq \delta
$$

for $\delta<n / 2$ and $r$ sufficiently large, then Cao and Zhou 4 showed that there exists some positive constant $C$ such that $\operatorname{Vol}\left(B_{x_{0}}(r)\right) \geq C r^{n-2 \delta}$. In 14 O. Munteanu and J. Wang proved the sharp result that every complete noncompact gradient shrinking Ricci soliton has at least linear volume growth, which answered the question asked by Cao and Zhou ([4, [2]) and Lei Ni whether a Calabi-Yau type lower volume growth estimate holds complete noncompact gradient shrinking Ricci solitons.

Theorem A (Munteanu and Wang [14]). Let $(M, g, f)$ be a complete noncompact gradient shrinking Ricci soliton. Then for any $x_{0} \in M$ there exists a constant $C>0$ such that

$$
\operatorname{Vol}\left(B_{x_{0}}(r)\right) \geq C r, \quad \text { for all } r>0,
$$

where $B_{x_{0}}(r)$ is the geodesic ball of $M$ of radius $r$ centered at $x_{0} \in M$.

For an $n$-dimensional complete noncompact self-shrinker $X: M^{n} \rightarrow \mathbb{R}^{n+m}$ satisfying

$$
H=-\frac{1}{2} X^{N}
$$

Lu Wang 17. proved that every entire graphical self-shrinker has polynomial volume growth. Then Q. Ding and Y. L. Xin 9] generalized it and showed that if the immersion is proper, then the self-shrinker has at most Euclidean volume growth. After that, Cheng and Zhou [7] improved Ding and Xin's result and gave a sharp volume growth estimate: they showed that $\operatorname{Vol}\left(B_{x_{0}}(r)\right) \leq C r^{n-2 \beta}$, with $\beta \leq \inf |H|^{2}$, where the ball $B_{x_{0}}(r)$ is defined by

$$
B_{x_{0}}(r)=\left\{x \in M: \rho_{x_{0}}(x)<r\right\}, \quad x_{0} \in M,
$$

with $\rho_{x_{0}}(x)=\left|X(x)-X\left(x_{0}\right)\right|$ is the extrinsic distance function.

In this paper, we consider the lower volume growth estimates for complete noncompact self-shrinkers, and an analogue of Munteanu-Wang's result will be proved.

Theorem 1.1. Let $X: M^{n} \rightarrow \mathbb{R}^{n+m}$ be a complete noncompact properly immersed self-shrinker. Then for any $x_{0} \in M$ there exists a constant $C>0$ such that

$$
\operatorname{Vol}\left(B_{x_{0}}(r)\right) \geq C r, \quad \text { for all } r>0,
$$

where the ball $B_{x_{0}}(r)$ is defined as in (1.5).

Remark 1.2. Note that this is sharp because the volume of the cylinder self-shrinker $X: \mathbb{S}^{n-1}(\sqrt{2(n-1)}) \times \mathbb{R} \rightarrow \mathbb{R}^{n+1}$ grows linearly. 


\section{Preliminaries}

For a complete immersed self-shrinker $X: M^{n} \rightarrow \mathbb{R}^{n+m}$ which satisfies (1.4), we have

$$
\begin{aligned}
|H|^{2}+\frac{1}{4} \Delta|X|^{2} & =\frac{n}{2}, \\
\nabla|X|^{2} & =2 X^{T} .
\end{aligned}
$$

Note that for a gradient shrinking Ricci soliton which satisfies (1.3), we take the trace in (1.3) and get

$$
R+\Delta f=\frac{n}{2}
$$

The main idea of this paper is to compare the two equations (2.1) and (2.3). In fact, we can correspond $|H|^{2}$ to $R$ and $\frac{1}{4}|X|^{2}$ to $f$, and then explore the similarities between the self-shrinker and the gradient shrinking Ricci soliton.

Denote $\rho(x)=|X|$; we have

$$
\nabla \rho=\frac{X^{T}}{|X|} \quad \text { and } \quad|\nabla \rho|=\frac{\left|X^{T}\right|}{|X|} \leq 1, \quad \text { for } \rho \geq 1 .
$$

Denote

$$
\begin{aligned}
& B(r)=\{x \in M: \rho(x)<r\} \\
& V(r)=\operatorname{Vol}(B(r))=\int_{B(r)} d v, \quad \eta(r)=\int_{B(r)}|H|^{2} d v .
\end{aligned}
$$

Recall that for a compact Riemannian manifold $(\Omega, g)$ with boundary and a function $f \in H^{1}(\Omega)$, we have the co-area formula (see [16, section 3.1])

$$
\int_{\Omega} h d v_{g}=\int_{-\infty}^{\infty} d s \int_{\{f=s\}} \frac{h}{|\nabla f|} d \sigma(s),
$$

which holds for any nonnegative function $h$ on $\Omega$. Here $d \sigma(s)$ is the area form on the set $\{f=s\}$ with respect to the induced metric from $(\Omega, g)$. Denote the set $\partial B(r)=\{x \in M: \rho(x)=r\}$. Since in the ball $B(r)$ the function $\rho(x)=|X(x)| \in$ $H^{1}(B(r))$ and $0 \leq \rho(x) \leq r$, from the above co-area formula we have

$$
\begin{aligned}
V(r) & =\int_{0}^{r} d s \int_{\{\rho=s\}} \frac{1}{|\nabla \rho|} d \sigma=\int_{0}^{r} d s \int_{\partial B(s)} \frac{1}{|\nabla \rho|} d \sigma \\
V^{\prime}(r) & =\int_{\partial B(r)} \frac{1}{|\nabla \rho|} d \sigma=r \int_{\partial B(r)} \frac{1}{\left|X^{T}\right|} d \sigma, \\
\eta(r) & =\int_{0}^{r} d s \int_{\partial B(s)} \frac{|H|^{2}}{|\nabla \rho|} d \sigma=\int_{0}^{r} s d s \int_{\partial B(s)} \frac{|H|^{2}}{\left|X^{T}\right|} d \sigma \\
\eta^{\prime}(r) & =r \int_{\partial B(r)} \frac{|H|^{2}}{\left|X^{T}\right|} d \sigma .
\end{aligned}
$$

In the above equations, for brevity we denote by $d \sigma$ the area form on the set $\partial B(s)$ which may depend on $s$. 
Now we state the following lemma:

Lemma 2.1. Let $X: M^{n} \rightarrow \mathbb{R}^{n+m}$ be a complete noncompact properly immersed self-shrinker. Then

$$
n V(r)-r V^{\prime}(r)=2 \eta(r)-\frac{4}{r} \eta^{\prime}(r) .
$$

Proof. Integrating (2.1) over $B(r)$, we have by using (1.4), (2.2), (2.4)-(2.10)

$$
\begin{aligned}
n V(r)-2 \int_{B(r)}|H|^{2} & =\frac{1}{2} \int_{B(r)} \Delta|X|^{2} d v \\
& =\frac{1}{2} \int_{\partial B(r)} \nabla|X|^{2} \cdot \nu d \sigma \\
& =\frac{1}{2} \int_{\partial B(r)} \nabla|X|^{2} \cdot \frac{\nabla \rho}{|\nabla \rho|} d \sigma \\
& =\int_{\partial B(r)}\left|X^{T}\right| d \sigma \\
& =\int_{\partial B(r)} \frac{|X|^{2}-4|H|^{2}}{\left|X^{T}\right|} d \sigma \\
& =r V^{\prime}(r)-4 \int_{\partial B(r)} \frac{|H|^{2}}{\left|X^{T}\right|} d \sigma .
\end{aligned}
$$

Remark 2.1. From the fourth equality in the above proof, we can get

$$
\frac{1}{V(r)} \int_{B(r)}|H|^{2} \leq \frac{n}{2}
$$

that is, the average of $|H|^{2}$ is bounded by $n / 2$.

Lemma 2.2. Let $X: M^{n} \rightarrow \mathbb{R}^{n+m}$ be a complete noncompact properly immersed self-shrinker. Then

$$
\frac{V\left(r_{1}\right)}{r_{1}^{n}}-\frac{V\left(r_{2}\right)}{r_{2}^{n}} \leq 2 n \frac{V\left(r_{1}\right)}{r_{1}^{n+2}}, \quad \text { for } r_{1}>r_{2} \geq r_{0}=\sqrt{2(n+2)} .
$$

Proof. Lemma 2.1 implies that

$$
\begin{aligned}
\left(r^{-n} V(r)\right)^{\prime} & =r^{-n-1}\left(r V^{\prime}(r)-n V(r)\right) \\
& =4 r^{-n-2} \eta^{\prime}(r)-2 r^{-n-1} \eta(r) .
\end{aligned}
$$

Integrating the above equation from $r_{2}$ to $r_{1}$, we get

$$
\begin{aligned}
r_{1}^{-n} V\left(r_{1}\right)-r_{2}^{-n} V\left(r_{2}\right)= & \int_{r_{2}}^{r_{1}} 4 s^{-n-2} \eta^{\prime}(s) d s-\int_{r_{2}}^{r_{1}} 2 s^{-n-1} \eta(s) d s \\
= & 4 r_{1}^{-n-2} \eta\left(r_{1}\right)-4 r_{2}^{-n-2} \eta\left(r_{2}\right) \\
& +2 \int_{r_{2}}^{r_{1}}\left(2(n+2)-s^{2}\right) s^{-n-3} \eta(s) d s .
\end{aligned}
$$

Choose $r_{0}=\sqrt{2(n+2)}$ and let $r_{1}>r_{2} \geq r_{0}$. Since $\eta(r)$ is nonnegative and nondecreasing in $r$, we have

$$
\begin{aligned}
\int_{r_{2}}^{r_{1}}\left(2(n+2)-s^{2}\right) s^{-n-3} \eta(s) d s & \leq \eta\left(r_{2}\right) \int_{r_{2}}^{r_{1}}\left(2(n+2)-s^{2}\right) s^{-n-3} d s \\
& \leq \eta\left(r_{2}\right)\left(-2 r_{1}^{-n-2}+2 r_{2}^{-n-2}\right) .
\end{aligned}
$$


Thus

$$
\begin{aligned}
r_{1}^{-n} V\left(r_{1}\right)-r_{2}^{-n} V\left(r_{2}\right) & \leq 4 r_{1}^{-n-2}\left(\eta\left(r_{1}\right)-\eta\left(r_{2}\right)\right) \\
& \leq 4 r_{1}^{-n-2} \eta\left(r_{1}\right) \\
& \leq 2 n r_{1}^{-n-2} V\left(r_{1}\right),
\end{aligned}
$$

where we used (2.12) in the last inequality. This completes the proof of Lemma 2.2.

Remark 2.2. Let $r_{2}=r_{0}$ and $r=r_{1}$ be sufficiently large in Lemma 2.2 ; we can obtain that

$$
V(r) \leq 2 r_{0}^{-n} V\left(r_{0}\right) r^{n} .
$$

Since $B_{x_{0}}(r) \subset B\left(r+\left|X_{0}\right|\right)$, we have

$$
\operatorname{Vol}\left(B_{x_{0}}(r)\right) \leq V\left(r+\left|X_{0}\right|\right) \leq C\left(r+\left|X_{0}\right|\right)^{n} \leq 2^{n} C r^{n}
$$

for $r \geq\left|X_{0}\right|$. This recovers Ding and Xin's result [9], which states that every complete noncompact properly immersed self-shrinker has at most Euclidean volume growth.

In the last part of this section, we recall the Logarithmic Sobolev inequality for submanifolds in Euclidean space; this was shown by K. Ecker in [10.

Proposition 2.1 (LSI). Let $X: M^{n} \rightarrow \mathbb{R}^{n+m}$ be an n-dimensional submanifold with measure $d v$. Then the inequality

$$
\begin{gathered}
\int_{M} f^{2}\left(\ln f^{2}\right) e^{-\frac{|X|^{2}}{4}} d v-\int_{M} f^{2} \ln \left(\int_{M} f^{2} e^{-\frac{|X|^{2}}{4}}\right) e^{-\frac{|X|^{2}}{4}} d v \\
\leq 2 \int_{M}|\nabla f|^{2} e^{-\frac{|X|^{2}}{4}} d v+\frac{1}{2} \int_{M}\left|H+\frac{1}{2} X^{N}\right|^{2} f^{2} e^{-\frac{|X|^{2}}{4}} d v \\
+C(n) \int_{M} f^{2} e^{-\frac{|X|^{2}}{4}}
\end{gathered}
$$

holds for any nonnegative function $f$ for which all integrals are well defined and finite, where $C(n)$ is a positive constant depending on $n$.

On the self-shrinker which satisfies (1.4), the Logarithmic Sobolev inequality (2.14) implies the following two inequalities:

(1) For any nonnegative function $f$ which satisfies the normalization

$$
\int_{M} f^{2} e^{-\frac{|X|^{2}}{4}} d v=1
$$

the inequality

$$
\int_{M} f^{2}(\ln f) e^{-\frac{|X|^{2}}{4}} d v \leq \int_{M}|\nabla f|^{2} e^{-\frac{|X|^{2}}{4}} d v+\frac{1}{2} C(n)
$$

holds.

(2) By substituting $f=u e^{\frac{|X|^{2}}{8}}$ into (2.14), the inequality

$$
\int_{M} u^{2} \ln u^{2}-\left(\int_{M} u^{2}\right)\left(\ln \int_{M} u^{2}\right) \leq 4 \int_{M}|\nabla u|^{2}+C(n) \int_{M} u^{2}
$$

holds for any nonnegative function $u$ for which all the integrals are well defined and finite. 


\section{Proof of Theorem 1.1}

In order to prove Theorem 1.1, we need the following lemma, which holds for any complete properly immersed submanifold in Euclidean space.

Lemma 3.1. Let $X: M^{n} \rightarrow \mathbb{R}^{n+m}$ be a complete properly immersed submanifold. For any $x_{0} \in M, r \leq 1$, if $|H| \leq \frac{C}{r}$ in $B_{x_{0}}(r)$ for some positive constant $C>0$, where the ball $B_{x_{0}}(r)$ is defined as in (1.5), then the following inequality holds:

$$
V_{x_{0}}(r)=\operatorname{Vol}\left(B_{x_{0}}(r)\right) \geq \kappa r^{n}
$$

here $\kappa=\omega_{n} e^{-C}$.

Proof. In $B_{x_{0}}(r)$ we have

$$
\begin{aligned}
\Delta \rho_{x_{0}}^{2}(x) & =2 n+2\left\langle X-X_{0}, H\right\rangle \\
& \geq 2 n-2|H| \rho_{x_{0}}(x) .
\end{aligned}
$$

If $|H| \leq \frac{C}{r}$ in $B_{x_{0}}(r)$, then in $B_{x_{0}}(r)$ we have

$$
2 n-2 \frac{C}{r} \rho_{x_{0}}(x) \leq \Delta \rho_{x_{0}}^{2}(x) .
$$

Integrating the above equation over $B_{x_{0}}(s)$ for $s \leq r$,

$$
\begin{aligned}
\left(2 n-2 \frac{C}{r} s\right) V_{x_{0}}(s) & \leq \int_{B_{x_{0}}(s)} \Delta \rho_{x_{0}}^{2}(x) \\
& =\int_{\partial B_{x_{0}}(s)} \nabla \rho_{x_{0}}^{2}(x) \cdot \nu \\
& =\int_{\partial B_{x_{0}}(s)} \nabla \rho_{x_{0}}^{2}(x) \cdot \frac{\nabla \rho_{x_{0}}(x)}{\left|\nabla \rho_{x_{0}}(x)\right|} \\
& =\int_{\partial B_{x_{0}}(s)} 2 \frac{\left|\left(X-X_{0}\right)^{T}\right|^{2}}{\left|\left(X-X_{0}\right)^{T}\right|} \\
& \leq 2 s \int_{\partial B_{x_{0}}(s)} \frac{\left|X-X_{0}\right|}{\left|\left(X-X_{0}\right)^{T}\right|} \\
& =2 s \int_{\partial B_{x_{0}}(s)} \frac{1}{\left|\nabla \rho_{x_{0}}\right|} \\
& =2 s V_{x_{0}}^{\prime}(s),
\end{aligned}
$$

where $\partial B_{x_{0}}(s)=\left\{x \in M: \rho_{x_{0}}(x)=s\right\}$ is the boundary of $B_{x_{0}}(s)$ and the last equality is due to the co-area formula. This implies

$$
\frac{V_{x_{0}}^{\prime}(s)}{V_{x_{0}}(s)} \geq \frac{n}{s}-\frac{C}{r}
$$

Integrating from $\epsilon>0$ to $r$, we have

$$
V_{x_{0}}(r) \geq \frac{V_{x_{0}}(\epsilon)}{\epsilon^{n}} r^{n} e^{-\frac{C}{r}(r-\epsilon)} .
$$

Let $\epsilon \rightarrow 0$; by $\lim _{\epsilon \rightarrow 0^{+}} \frac{V_{x_{0}}(\epsilon)}{\epsilon^{n}}=\omega_{n}$, we have

$$
V_{x_{0}}(r) \geq \kappa r^{n} \quad\left(\kappa=\omega_{n} e^{-C}\right) .
$$


Remark 3.1. As pointed out to us by Ovidiu Munteanu, Lemma 3.1 also follows from the Michael-Simon Sobolev inequality; see page 377 in [13].

Next we will prove that every complete noncompact properly immersed selfshrinker has infinite volume. The argument in the following proof is an adoption of Cao and Zhu's 2 proof that complete noncompact shrinking Ricci solitons have infinite volume, following an idea in Perelman's proof on the uniform diameter estimate for the normalized Kähler-Ricci flow on Fano manifolds. See also the proof of no local collapsing in Theorem 3.3.3 in [5].

Lemma 3.2. Every complete noncompact properly immersed self-shrinker $X$ : $M^{n} \rightarrow \mathbb{R}^{n+m}$ has infinite volume.

Proof. We are going to show that if $M$ has finite volume, then we shall obtain a contradiction to the Logarithmic Sobolev inequality (2.15). We denote the annulus region

$$
A\left(k_{1}, k_{2}\right)=\left\{x \in M: 2^{k_{1}} \leq \rho(x) \leq 2^{k_{2}}\right\}, \quad V\left(k_{1}, k_{2}\right)=\operatorname{Vol}\left(A\left(k_{1}, k_{2}\right)\right) ;
$$

here $\rho(x)=|X|$. Since $X: M^{n} \rightarrow \mathbb{R}^{n+m}$ is complete noncompact properly immersed, $X(M)$ cannot be contained in a compact Euclidean ball $\bar{B}(R)$ with radius $R<+\infty$. Then for $k$ large enough, $A(k, k+1)$ contains at least $2^{2 k-1}$ disjoint balls

$$
B_{x_{i}}(r)=\left\{x \in M, \rho_{x_{i}}(x)<r\right\}, \quad x_{i} \in M, r=2^{-k},
$$

where $\rho_{x_{i}}(x)=\left|X(x)-X\left(x_{i}\right)\right|$ is the extrinsic distance function. Note that on the self-shrinker

$$
|H|=\frac{1}{2}\left|X^{N}\right| \leq \frac{1}{2}|X| \leq 2^{k}=\frac{1}{r}, \quad \text { in } A(k, k+1) .
$$

Thus by Lemma 3.1, each ball $B_{x_{i}}(r)$ has at least volume $\kappa 2^{-k n}$. Here $\kappa=\omega_{n} e^{-1}$. So we have

$$
V(k, k+1) \geq \kappa 2^{2 k-1} 2^{-k n} .
$$

Suppose that $\operatorname{Vol}(M)<+\infty$. Then for every $\epsilon>0$, there exists a large constant $k_{0}>0$ such that if $k_{2}>k_{1}>k_{0}$, we have

$$
V\left(k_{1}, k_{2}\right) \leq \epsilon
$$

and we can also choose $k_{1}, k_{2}$ in such a way that

$$
V\left(k_{1}, k_{2}\right) \leq 2^{4 n} V\left(k_{1}+2, k_{2}-2\right) .
$$

Indeed, we may first choose $K>0$ sufficiently large, and let $k_{1} \approx K / 2, k_{2} \approx$ $3 K / 2$. Suppose (3.8) does not hold, i.e.,

$$
V\left(k_{1}, k_{2}\right) \geq 2^{4 n} V\left(k_{1}+2, k_{2}-2\right) .
$$

If

$$
V\left(k_{1}+2, k_{2}-2\right) \leq 2^{4 n} V\left(k_{1}+4, k_{2}-4\right),
$$

then we are done; otherwise we can repeat this process. After $j$ steps we get

$$
V\left(k_{1}, k_{2}\right) \geq 2^{4 n j} V\left(k_{1}+2 j, k_{2}-2 j\right) .
$$

When $j \approx K / 4$, (3.6) implies that

$$
\operatorname{Vol}(M) \geq V\left(k_{1}, k_{2}\right) \geq 2^{n K} V(K, K+1) \geq \kappa 2^{2 K-1} .
$$


But we have already assumed $\operatorname{Vol}(M)$ is finite, so after finitely many steps (3.8) must hold for some $k_{2}>k_{1}$. Thus for any $\epsilon>0$ we can choose $k_{1}$ and $k_{2} \approx 3 k_{1}$ such that both (3.7) and (3.8) are valid.

Now we are going to derive a contradiction to the Logarithmic Sobolev inequality (2.15). We define a smooth cut-off function $\psi(t)$ by

$$
\psi(t)=\left\{\begin{array}{ll}
1, & 2^{k_{1}+2} \leq t \leq 2^{k_{2}-2}, \\
0, & \text { outside }\left[2^{k_{1}}, 2^{k_{2}}\right],
\end{array} \quad 0 \leq \psi(t) \leq 1, \quad\left|\psi^{\prime}(t)\right| \leq 1 .\right.
$$

Then let

$$
f(x)=e^{L+\frac{|X|^{2}}{8}} \psi(\rho(x))
$$

we can choose $L$ such that

$$
1=\int_{M} f^{2} e^{-\frac{|X|^{2}}{4}}=e^{2 L} \int_{A\left(k_{1}, k_{2}\right)} \psi^{2}(\rho(x)) .
$$

By the Logarithmic Sobolev inequality (2.15) we have

$$
\begin{aligned}
\frac{1}{2} C(n) \geq & \int_{A\left(k_{1}, k_{2}\right)} e^{2 L} \psi^{2}\left(L+\frac{|X|^{2}}{8}+\ln \psi\right) \\
& -\int_{A\left(k_{1}, k_{2}\right)} e^{2 L}\left|\psi^{\prime} \nabla \rho+\psi \frac{X^{T}}{4}\right|^{2} \\
\geq & \int_{A\left(k_{1}, k_{2}\right)} e^{2 L} \psi^{2}\left(L+\frac{|X|^{2}}{8}+\ln \psi\right) \\
& -2 \int_{A\left(k_{1}, k_{2}\right)} e^{2 L}\left|\psi^{\prime}\right|^{2}-\frac{1}{8} \int_{A\left(k_{1}, k_{2}\right)} e^{2 L} \psi^{2}|X|^{2} \\
= & L+\int_{A\left(k_{1}, k_{2}\right)} e^{2 L} \psi^{2} \ln \psi-2 \int_{A\left(k_{1}, k_{2}\right)} e^{2 L}\left|\psi^{\prime}\right|^{2} \\
\geq & L-\left(\frac{1}{2 e}+2\right) e^{2 L} V\left(k_{1}, k_{2}\right),
\end{aligned}
$$

where we have used $|\nabla \rho(x)| \leq 1$ and the elementary inequality $t \ln t \geq-\frac{1}{e}$ for $0 \leq t \leq 1$. Then (3.8) implies

$$
\begin{aligned}
\frac{1}{2} C(n) & \geq L-\left(\frac{1}{2 e}+2\right) e^{2 L} 2^{4 n} V\left(k_{1}+2, k_{2}-2\right) \\
& \geq L-\left(\frac{1}{2 e}+2\right) 2^{4 n} e^{2 L} \int_{A\left(k_{1}, k_{2}\right)} \psi^{2}(\rho(x)) \\
& =L-\left(\frac{1}{2 e}+2\right) 2^{4 n},
\end{aligned}
$$

where the last equality is due to (3.9). On the other hand, by (3.7), (3.9) and $0 \leq \psi \leq 1$, we have

$$
1 \leq e^{2 L} \epsilon
$$

So we can make $L$ arbitrarily large by letting $\epsilon>0$ be sufficiently small. This contradicts (3.10) because $C(n)$ is just a universal positive constant depending on $n$. Therefore $M$ must have infinite volume.

Remark 3.2. In [7, Xu Cheng and Detang Zhou proved that if the self-shrinker is not properly immersed, then it must also have infinite volume. 
Now we are ready to prove Theorem 1.1.

Proof of Theorem 1.1. We use the similar arguments of Munteanu and Wang in their proof of Theorem A. First we can choose $c>0$ such that $V(r)>0$ for $r \geq c$. To prove Theorem 1.1, it suffices to show that there exists a constant $C>0$ depending only on $n$ such that

$$
V(r) \geq C r
$$

holds for all $r \geq c$. Indeed, if (3.12) holds, then $\forall x_{0} \in M$, since for $r$ sufficiently large,

$$
B_{x_{0}}(r) \supset B\left(r-\left|X_{0}\right|\right),
$$

this implies

$$
V_{x_{0}}(r) \geq V\left(r-\left|X_{0}\right|\right) \geq C\left(r-\left|X_{0}\right|\right) \geq \frac{C}{2} r
$$

for $r \geq 2\left|X_{0}\right|$.

Now we are going to prove (3.12) by contradiction. Assume that for any $\epsilon>0$, there exists $r \geq c$ such that

$$
V(r) \leq \epsilon r
$$

Without loss of generality, we can assume $r \in \mathbb{N}$ and consider the following set:

$$
D:=\{k \in \mathbb{N}: V(t) \leq 2 \epsilon t \text { for all integers } r \leq t \leq k\} .
$$

Obviously $D$ is nonempty because $r \in D$; we want to prove that any integer $k \geq r$ is in $D$.

For $t \geq c$, we define a function $u$ by

$$
u(x)=\left\{\begin{array}{cl}
1 & \text { in } B(t+1) \backslash B(t), \\
t+2-\rho(x) & \text { in } B(t+2) \backslash B(t+1), \\
\rho(x)-(t-1) & \text { in } B(t) \backslash B(t-1), \\
0 & \text { otherwise. }
\end{array}\right.
$$

Substituting $u(x)$ into the Logarithmic Sobolev inequality (2.16), we obtain

$$
-\left(\int_{M} u^{2}\right) \ln (V(t+2)-V(t-1)) \leq C_{0}(V(t+2)-V(t-1))
$$

with $C_{0}=C(n)+4+\frac{1}{e}$. Here we have used $|\nabla \rho(x)| \leq 1$ and the elementary inequality $t \ln t \geq-\frac{1}{e}$ for $0 \leq t \leq 1$.

From Lemma 2.2, we have

$$
\frac{V(t+1)}{(t+1)^{n}}-\frac{V(t)}{t^{n}} \leq 2 n \frac{V(t+1)}{(t+1)^{n+2}}, \quad \text { for } t \geq \sqrt{2(n+2)}
$$

Then

$$
V(t+1) \leq V(t) \frac{(t+1)^{n}}{t^{n}}\left(1-\frac{2 n}{(t+1)^{2}}\right)^{-1}
$$


This implies for $t$ sufficiently large,

$$
\begin{aligned}
V(t+1)-V(t) & \leq V(t)\left(\frac{(t+1)^{n}}{t^{n}}\left(1+\frac{2 n}{(t+1)^{2}}+O\left(\frac{1}{(t+1)^{4}}\right)\right)-1\right) \\
& \leq V(t)\left(\left(1+\frac{1}{t}\right)^{n}-1+\frac{C}{t^{2}}\left(1+\frac{1}{t}\right)^{n-2}\right) \\
& \leq V(t) \frac{C}{t} .
\end{aligned}
$$

So there exists some constant $C_{1}(n)$ such that for all $t \geq C_{1}(n)$,

$$
\begin{aligned}
V(t+1)-V(t) & \leq \tilde{C}_{1} \frac{V(t)}{t} \text { and } \\
V(t+1) & \leq 2 V(t),
\end{aligned}
$$

where $\tilde{C}_{1}$ depends only on $n$. Combining (3.18) and (3.19) gives that for all $t \geq$ $C_{1}(n)+1$,

$$
\begin{aligned}
V(t+2)-V(t-1) & \leq \tilde{C}_{1}\left(\frac{V(t+1)}{t+1}+\frac{V(t)}{t}+\frac{V(t-1)}{t-1}\right) \\
& \leq \tilde{C}_{1}\left(\frac{2}{t+1}+\frac{1}{t}+\frac{1}{t}\left(1+\frac{1}{C_{1}(n)}\right)\right) V(t) \\
& \leq C_{2} \frac{V(t)}{t},
\end{aligned}
$$

where $C_{2}$ depends only on $n$. Note that we can assume $r \geq C_{1}(n)+1$ for the $r$ satisfying (3.14). In fact, if for any given $\epsilon>0$, all the $r$ which satisfy (3.14) are bounded above by $C_{1}(n)+1$, then $V(r) \geq \epsilon r$ holds for any $r>C_{1}(n)+1$. This implies that $M$ has at least linear volume growth.

Then for all integers $r \leq t \leq k$, we have $t \in D$, and (3.20) implies

$$
V(t+2)-V(t-1) \leq 2 C_{2} \epsilon .
$$

If we choose $\epsilon$ such that $2 C_{2} \epsilon<1$, and noting that

$$
\int_{M} u^{2} \geq V(t+1)-V(t)
$$

(3.16) then implies

$$
(V(t+1)-V(t)) \ln \left(2 C_{2} \epsilon\right)^{-1} \leq C_{0}(V(t+2)-V(t-1)) .
$$

Iterating from $t=r$ to $t=k$ and summing up give that

$$
(V(k+1)-V(r)) \ln \left(2 C_{2} \epsilon\right)^{-1} \leq 3 C_{0} V(k+2) \leq 6 C_{0} V(k+1),
$$

where we used (3.19) in the last inequality. Therefore

$$
\begin{aligned}
V(k+1) & \leq V(r) \frac{\ln \left(2 C_{2} \epsilon\right)^{-1}}{\ln \left(2 C_{2} \epsilon\right)^{-1}-6 C_{0}} \\
& \leq \epsilon r \frac{\ln \left(2 C_{2} \epsilon\right)^{-1}}{\ln \left(2 C_{2} \epsilon\right)^{-1}-6 C_{0}} .
\end{aligned}
$$

We can choose $\epsilon$ small enough such that

$$
\frac{\ln \left(2 C_{2} \epsilon\right)^{-1}}{\ln \left(2 C_{2} \epsilon\right)^{-1}-6 C_{0}} \leq 2 .
$$


So (3.25) implies

$$
V(k+1) \leq 2 \epsilon r, \quad \text { for any } k \in D .
$$

Noting that $r \leq k+1$, (3.27) implies $k+1 \in D$. Then by induction we conclude that $D$ contains all the integers $k \geq r$. However, (3.27) implies

$$
V(k) \leq 2 \epsilon r, \quad \text { for any integer } k \geq r .
$$

This implies that $M$ has finite volume, which contradicts Lemma 3.2. So there exists no such $r>c$ such that $V(r) \leq \epsilon r$ with $\epsilon>0$ chosen in (3.26). That is, $V(r) \geq \epsilon r$ for $r>c$, and this completes the proof of Theorem 1.1.

By assuming some condition on $|H|^{2}$, we can further prove the following result.

Proposition 3.1. Let $X: M^{n} \rightarrow \mathbb{R}^{n+m}$ be a complete properly immersed selfshrinker. Suppose the average norm square of the mean curvature satisfies the upper bound

$$
\frac{1}{\operatorname{Vol}(B(r))} \int_{B(r)}|H|^{2} \leq \delta
$$

for some $\delta<\frac{n}{2}$ and $r$ sufficiently large. Then for any $x_{0} \in M$, there exists some positive constant $C$ such that

$$
\operatorname{Vol}\left(B_{x_{0}}(r)\right) \geq C r^{n-2 \delta} .
$$

Proof. Combining the assumption (3.28) with Lemma 2.1 gives that

$$
(n-2 \delta) V(r) \leq r V^{\prime}(r)
$$

then

$$
\frac{V^{\prime}(r)}{V(r)} \geq \frac{n-2 \delta}{r}
$$

Integrating from 1 to $r$ gives

$$
V(r) \geq V(1) r^{n-2 \delta}
$$

Since $\operatorname{Vol}\left(B_{x_{0}}(r)\right) \geq V\left(r-\left|X_{0}\right|\right)$ for $r>\left|X_{0}\right|$, we have

$$
\operatorname{Vol}\left(B_{x_{0}}(r)\right) \geq V(1)\left(r-\left|X_{0}\right|\right)^{n-2 \delta} \geq\left(\frac{1}{2}\right)^{n-2 \delta} V(1) r^{n-2 \delta}
$$

for $r>2\left|X_{0}\right|$.

\section{ACKNOWLEDGMENTS}

The authors would like to thank Professors Huai-Dong Cao, Ovidiu Munteanu and Detang Zhou for their interest and useful comments regarding their work. The authors thank Robert Haslhofer for informing them of the paper [14] by Ovidiu Munteanu and Jiaping Wang. They also thank the referee for suggestions which made the paper more readable. 


\section{REFERENCES}

[1] E. Calabi, On manifolds with non-negative Ricci-curvature II, Notices Amer. Math. Soc. 22 (1975), A205

[2] Huai-Dong Cao, Geometry of complete gradient shrinking Ricci solitons, Geometry and analysis. No. 1, Adv. Lect. Math. (ALM), vol. 17, Int. Press, Somerville, MA, 2011, pp. 227-246. MR2882424

[3] Huai-Dong Cao and Haizhong Li, A gap theorem for self-shrinkers of the mean curvature flow in arbitrary codimension, Calc. Var. Partial Differential Equations 46 (2013), no. 3-4, 879-889, DOI 10.1007/s00526-012-0508-1. MR3018176

[4] Huai-Dong Cao and Detang Zhou, On complete gradient shrinking Ricci solitons, J. Differential Geom. 85 (2010), no. 2, 175-185. MR2732975 (2011k:53040)

[5] Huai-Dong Cao and Xi-Ping Zhu, A complete proof of the Poincaré and geometrization conjectures - application of the Hamilton-Perelman theory of the Ricci flow, Asian J. Math. 10 (2006), no. 2, 165-492. MR2233789 (2008d:53090)

[6] J. Carrillo and L. Ni, Sharp logarithmic Sobolev inequalities on gradient solitons and applications, Comm. Anal. Geom. 17 (2009), 721-753. MR.3010626

[7] Xu Cheng and Detang Zhou, Volume estimate about shrinkers, Proc. Amer. Math. Soc. 141 (2013), no. 2, 687-696, DOI 10.1090/S0002-9939-2012-11922-7. MR2996973

[8] Tobias H. Colding and William P. Minicozzi II, Generic mean curvature flow I: generic singularities, Ann. of Math. (2) 175 (2012), no. 2, 755-833, DOI 10.4007/annals.2012.175.2.7. MR2993752

[9] Q. Ding and Y. L. Xin, Volume growth, eigenvalue and compactness for self-shrinkers, Asian J. Math. 17 (2013), no. 3, 443-456. MR3119795

[10] Klaus Ecker, Logarithmic Sobolev inequalities on submanifolds of Euclidean space, J. Reine Angew. Math. 522 (2000), 105-118, DOI 10.1515/crll.2000.033. MR1758578 (2001h:58035)

[11] Gerhard Huisken, Asymptotic behavior for singularities of the mean curvature flow, J. Differential Geom. 31 (1990), no. 1, 285-299. MR1030675 (90m:53016)

[12] Peter Li, Lecture notes on geometric analysis, Lecture Notes Series, vol. 6, Seoul National University Research Institute of Mathematics Global Analysis Research Center, Seoul, 1993. MR 1320504 (96m:58269)

[13] J. H. Michael and L. M. Simon, Sobolev and mean-value inequalities on generalized submanifolds of $R^{n}$, Comm. Pure Appl. Math. 26 (1973), 361-379. MR0344978 (49 \#9717)

[14] Ovidiu Munteanu and Jiaping Wang, Analysis of weighted Laplacian and applications to Ricci solitons, Comm. Anal. Geom. 20 (2012), no. 1, 55-94. MR2903101

[15] G. Perelman, The entropy formula for the Ricci flow and its geometric applications, arXiv: math. DG/0211159

[16] R. Schoen and S.-T. Yau, Lectures on differential geometry, Conference Proceedings and Lecture Notes in Geometry and Topology, I, International Press, Cambridge, MA, 1994. Lecture notes prepared by Wei Yue Ding, Kung Ching Chang [Gong Qing Zhang], Jia Qing Zhong and Yi Chao $\mathrm{Xu}$; translated from the Chinese by Ding and S. Y. Cheng; preface translated from the Chinese by Kaising Tso. MR 1333601 (97d:53001)

[17] Lu Wang, A Bernstein type theorem for self-similar shrinkers, Geom. Dedicata 151 (2011), 297-303, DOI 10.1007/s10711-010-9535-2. MR2780753(2012e:53130)

[18] Shing Tung Yau, Some function-theoretic properties of complete Riemannian manifold and their applications to geometry, Indiana Univ. Math. J. 25 (1976), no. 7, 659-670. MR0417452 (54 \#5502)

[19] Shi Jin Zhang, On a sharp volume estimate for gradient Ricci solitons with scalar curvature bounded below, Acta Math. Sin. (Engl. Ser.) 27 (2011), no. 5, 871-882. MR2786449 (2012g:53081)

Department of Mathematical Sciences, and Mathematical Sciences Center, Tsinghua University, 100084, Beijing, People's Republic of China

E-mail address: hli@math.tsinghua.edu.cn

Department of Mathematical Sciences, Tsinghua University, 100084, Beijing, PeoPLE'S REPUBLIC OF CHINA

E-mail address: wei-y09@mails.tsinghua.edu.cn 